\title{
Optimisation of the undercut level elevation in block caving mines using a mathematical programming framework
}

\author{
R Noriega University of Alberta, Canada \\ Y Pourrahimian University of Alberta, Canada \\ WL Victor University of Alberta, Canada
}

\begin{abstract}
The selection of an elevation for the placement of the undercut level is a decisive initial step in the planning and design of block caving mines. It is key for the success of a caving project to define the undercut elevation that will most likely yield the highest possible net present value (NPV) of the operation, considering the discounting periods from both the vertical draw rate extraction of the ore and the horizontal mining direction advancement.

This paper outlines a mathematical programming framework to determine the best undercut elevation by formulating a simplified linear integer programming (IP) model that captures the discounting of the profits from the vertical draw rate extraction and the horizontal advance direction that can be applied at the early stages of a caving project. The IP model comprises a simplified block caving scheduling algorithm that considers 'mining units' (MU), which are groupings of blocks within each column based on the minimum draw rate condition and the undercut elevation considered. The formulation considers the following constraints: mining capacity, minimum and maximum draw rate, vertical precedence within columns and a horizontal precedence based on a concave mining advancement front. The model is set on an iterative loop over the different possible levels.
\end{abstract}

The model is tested on a case study, where it provides a tool to evaluate the potential mineable reserves, define the optimal undercut elevation, and a starting schedule for future detailed engineering design.

Keywords: block caving, undercut elevation, optimisation, integer programming

\section{Introduction}

One of the initial steps in the long-term planning of block caving mines is the selection of an undercut elevation and the definition of a mining footprint, after which a drawpoint layout is built, and the production schedule is generated (Diering 2000; Rubio 2002). The selection of a specific undercut elevation constrains the downstream planning process, having a direct influence in the potential net present value (NPV) of the project, and can be thought of as an optimisation problem on its own.

The current industry standard for the selection of an undercut elevation is the Footprint Finder module in GEMS PCBC software (Dassault Systèmes 2018). As explained by (Diering et al. 2008), Footprint Finder evaluates the different levels considering each block within a specific level as a drawpoint from which the discounted economic value of the column above is calculated, with the capability of applying Laubscher's vertical mixing model (Laubscher 1994). The level's economic value is then obtained as the sum of each column within. A method described by (Elkington 2012) finds the optimal cave outline for a given undercut elevation that maximises the metal content, by following an approach similar to that of pit outline optimisation, using an integer programming (IP) method considering operational constraints. This method works at a block level scale and provides an approximation to the potential final cave shape for a given elevation. However, it does not involve economic parameters. Another proposed method (Vargas et al. 2014) considers the caving outline definition as an inversed ultimate pit problem, and for each level, calculates the 
vertically discounted economic value for the caving envelope considering precedence constraints on the inverted block model.

The economic value of the extracted ore is subject to vertical discounting on each individual column, as each column is opened at a certain period based on the mining direction and the starting point in the footprint. The starting point and direction of mining are also usually a geotechnical decision, as it is desirable to start extraction in weaker rocks to achieve the hydraulic radius required and steady production rates early in the mine life (Bartlett 1992; Tukker et al. 2016). Therefore, the horizontal discounting due to the undercut layout sequence has to be considered in order to evaluate and select the best possible undercut elevation. The sequencing and scheduling problem in block caving is a complex optimisation problem that involves millions of possible combinations for a given set of drawpoints on a specific undercut level (Khodayari \& Pourrahimian 2015). While the use of mathematical programming techniques can guarantee an optimal solution for block caving sequencing and scheduling (Chanda 1990; Pourrahimian \& Askari-Nasab 2014; Pourrahimian et al. 2012, 2013; Rahal et al. 2008), it is often at the expense of large and prohibitive processing times.

The following method provides a simplified scheduling IP formulation applicable at the block model scale that can be repeated over multiple levels at reasonable processing times, to find the optimal undercut elevation for block caving mines considering time discounting over the vertical direction within each column, and the horizontal advancement direction.

Two assumptions are made to simplify the schedule optimisation problem that are in line with block caving operational practices. The first assumption is that instead of considering each individual block as a single drawpoint, a grouping of individual adjacent blocks is performed to represent a draw column or drawzone. Within each drawzone, the individual blocks are then vertically aggregated into representative mining units based on the minimum draw rate required. These mining units are then scheduled based on a concave horizontal mining advancement front, which is desirable to provide a better control of structures and a more secure undercutting (Brown 2002), and mining capacity constraints to evaluate the economic potential of each undercut level. These assumptions greatly reduce the number of variables and possible combinations while still being representative of the extraction practice in block caving operations, and provide some useful insight for the planning process at the early stages of the project.

\section{Method}

The first step is to generate the projected draw column areas on the undercut footprint by grouping the individual block model columns, to generate a more representative scenario of the ore extraction in block caving mines where the extraction is carried from draw columns over drawpoints at the undercut level. The representative columns are selected in order to account for the potential drawpoint layout geometry configurations, such as the different spacings between the drawpoints and the minimum required hydraulic radius to sustain mining (Malaki et al. 2017). These are selected on a case-by-case basis depending on the properties of the rock mass.

Figure 1 shows a schematic representation of the aggregation of multiple individual block model columns into a single representative draw column for the purpose of this model. This zone is parameterised by two dimensions, a size in $\mathrm{X}$ and a size in $\mathrm{Y}$, to define a rectangular area of influence in order to use the block model information for calculation of tonnages and metal content within it. The dimensions of this representative column can be adjusted to match the required spacing between drawpoints ( $A$ and $B$ in Figure 1 ) but is constrained by the dimensions of the underlying block model. 


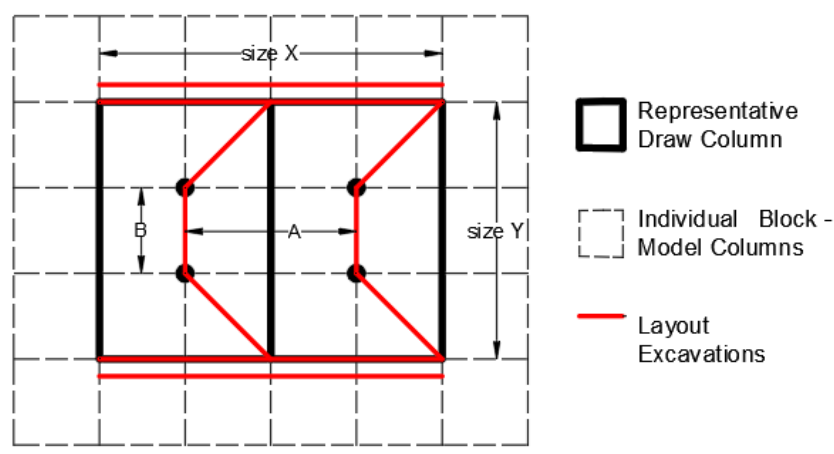

Figure 1 Schematic of the draw column aggregation from the individual block model columns at any undercut level

The grouping of the columns into the representative units for a given undercut is then an optimisation problem on its own, and its solution can provide useful insight on the drawpoint layout design, and a systematic way to evaluate different levels automatically. To optimise the layout, the key parameter considered is to maximise the total metal content of the units. The metal content was selected to not include the economic parameters yet, but still guarantee a priority on the selection of high-grade areas. The metal content of each unit is calculated as the summation of the metal contents of each individual block model columns that are part of it. Each column is considered as continuous, and the metal summation is carried until a first block of waste is found or a specific maximum column height is reached, to avoid waste material within the cave profile and to consider the operational maximum draw height.

Based on the representative unit dimensions in $X$ and $Y$, all possible units are generated on a given undercut elevation plan view and the associated total metal content for each representative column is calculated as described above.

The optimisation problem is then to find the combination of column units that yield the highest metal content from the deposit. The optimal column units must be adjacent and not include units with individual columns that do not the meet a minimum height operational constraint. To achieve this, all column units that do not meet the operational criteria are assigned a 0 metal content value. The optimisation model includes a constraint to avoid overlapping. This initial IP optimisation model is described below.

Indices

$p \in\{1, \ldots, P\}$

$b \in\{1, \ldots, B\}$

Index for all possible column units.

Index for all possible ore blocks in a given undercut level.

Sets

$O^{b}$

Decision variables

$x_{p} \in\{0,1\}$

$y_{b} \in\{0,1\}$

Parameters

$M_{p}$

Objective function
Set containing all column units that overlap with block $b$, with number of elements $N\left(O^{b}\right)$.

Binary variable controlling the decision to include unit $p$ in the layout.

Binary variable used to ensure that all ore blocks in the undercut are covered with no gaps in the layout.

Metal content of column unit $p$.

$$
\operatorname{Max} \sum_{1}^{P} M_{p} \times x_{p}
$$


Constraints

$$
\begin{gathered}
\sum_{1}^{N\left(O^{b}\right)} x_{p} \leq y_{b} \quad \forall b \in\{1, \ldots, B\} \\
y_{b}=1 \quad \forall b \in\{1, \ldots, B\}
\end{gathered}
$$

Equation 1 represents the objective function to maximise the metal content of the selected units, with the consideration that units with any individual column that does not meet the minimum column height (continuous ore from the undercut) are assigned a 0 value. Equations 2 and 3 represent the overlapping constraint to assure that only one column unit from all those that share at least a single block is selected, while also guaranteeing that all ore blocks in the undercut level are covered to avoid gaps in the layout.

After this initial step, a layout is generated for any given undercut, which simplifies the number of variables involved for the next scheduling optimisation step while still being representative of the operating conditions in block caving operations.

The second assumption mentioned above is the grouping of block slices along the vertical section of each of the column units based on the minimum draw rate. The decision to group the blocks on these mining units is based on the condition that, on each period, at least the minimum tonnes, or inches, have to be drawn continuously from each unit. The tonnage of each mining unit is the summation of the tonnage of the individual blocks within it, and the grade of the mining unit is the tonnage-weighted average grade of the individual blocks. As observed in Figure 1, this minimum draw rate would apply to each pair of drawpoints on the same excavation, and extracting ore from the same draw zone. While resolution is lost for the scheduling along the vertical section of each column unit due to the grouping, it further reduces the number of variables and possible combinations to achieve an optimal solution for the scheduling problem under reasonable processing times.

The simplified IP model is then formulated to maximise the discounted profit, or NPV, of the extraction of each mining units over a number of periods. The model is constrained by the total mining capacity, the maximum draw rate from each column unit, the vertical precedence within each column and the horizontal precedence across the layout based on a concave advancement front.

Indices

$$
\begin{array}{ll}
u \in\{1, \ldots, U\} & \text { Index for all mining units. } \\
t \in\{1, \ldots, T\} & \text { Index for all periods. } \\
c \in\{1, \ldots, C\} & \text { Index for all column units. }
\end{array}
$$

Sets

$C^{c}$

$V^{u, c}$

$H^{u, c}$

Decision variables

$z_{u, c}^{t} \in\{0,1\}$

\section{Parameters}

$$
P_{u, c}^{t}
$$

$\bar{M}$
Set containing the mining units that are within column unit $c$. Each set has a total number of elements $C_{c}$.

Single element set containing the mining unit directly below unit $u$ of column $c$.

Set containing the column unit that has to be opened previous to the extraction of unit $u$ of column $c$ based on a horizontal concave mining direction. Each set has a total number of elements $H_{u}$.

Binary variable controlling the decision to extract mining unit $u$ of column $c$ on period $t$.

Discounted profit for the extraction of unit $u$ of column $c$ in period $t$.

Maximum mining capacity (tonnes/period). 
Minimum mining capacity (tonnes/period).

$T_{u}$

Tonnage of mining unit $u$.

$\overline{D R}$

Maximum draw rate from each column unit (tonnes/period).

$\underline{D R}$

Minimum draw rate from each column unit (tonnes/period).

Objective function

$$
\operatorname{Max} \sum_{1}^{T} \sum_{1}^{P} P_{u, c}^{t} \times z_{u, c}^{t}
$$

Constraints

$$
\begin{gathered}
M \leq \sum_{1}^{P} T_{u} \times z_{u, c}^{t} \leq \bar{M} \quad \forall t \in\{1, \ldots, T\} \quad \forall c \in\{1, \ldots, C\} \\
\sum_{1}^{T} z_{u, c}^{t} \leq 1 \quad \forall u \in\{1, \ldots, U\} \forall c \in\{1, \ldots, C\} \\
z_{u, c}^{t} \leq z_{V}^{t} u, c+z_{V}^{t-1} \quad \forall u \in\{2, \ldots, U\} \quad \forall t \in\{2, \ldots, T\} \forall c \in\{1, \ldots, C\} \\
\sum_{1}^{C_{c}} T_{u, c} \times z_{u, c}^{t} \leq \overline{D R} \quad \forall u \in\{1, \ldots, U\} \quad \forall t \in\{1, \ldots, T\} \forall c \in\{1, \ldots, C\} \\
H_{u} \times z_{1, c}^{t} \leq \sum_{1}^{T} \sum_{1}^{H_{u}} z_{1, c}^{t} \quad \forall c \in\{1, \ldots, C\} \quad \forall t \in\{1, \ldots, T\} \forall c \in\{1, \ldots, C\}
\end{gathered}
$$

Equation 4 shows the objective function of the optimisation model that maximises the total NPV of the operation by considering the discounted profit from the extraction of each block. The profit is calculated as the difference between the revenues and the costs associated to mining each block and is then discounted based on the period of extraction. Equation 5 corresponds to the mining capacity constraint, and ensures that the total ore tonnage extracted from all columns over each period is within the minimum and maximum established ranges. Equation 6 guarantees that no mining unit is extracted more than once. Equation 7 establishes the vertical precedence within mining units of the same column, and guarantees that to extract a unit, the unit directly below it has to be extracted in the same or previous period to assure a continuous draw of the column. Equation 8 ensures that the total ore tonnage extracted from each column unit on each period is less than or equal to the maximum draw rate. Since the mining units are built based on the minimum draw rate volume, there is no need to include a minimum draw rate constraint. Finally, Equation 9 forces the horizontal precedence constraint. The horizontal precedence constraint is based on a concave mining advancement front and is defined by three angles - the azimuth of the main advancement direction $a$, and the two diagonal angles $a 1$ and $a 2$ (Figure 2). The constraints guarantee that a column unit can initiate extraction only after all of the directly preceding columns, based on the mentioned direction, have started extraction.

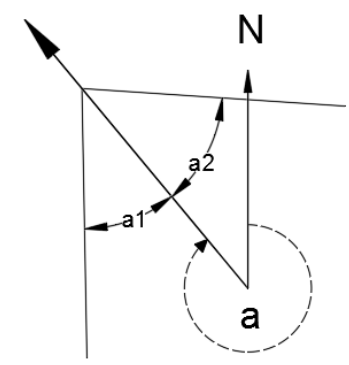

Figure 2 Concave advancement from for the establishment of horizontal precedence constraints in the optimisation model

The optimisation framework then ensures that, for a given undercut elevation, the column unit layout that maximises the metal content is found first, then the mining units are built based on the optimised layout and the minimum draw rate to be scheduled considering the vertical and horizontal profit discounting, and operating constraints as well. The process is then carried over the different possible levels of the deposit to select the level that yields the highest NPV. The model then can be used to individually evaluate multiple scenarios, such as different mining directions or operational parameters, on any specific undercut level of interest. 


\section{Case study}

The optimisation model was tested on a copper deposit case study. A block model of the deposit is built with blocks of $10 \times 10 \times 10 \mathrm{~m}$ (Figure 3). The deposit has a total of $58.5 \mathrm{M} \mathrm{t}$ of ore with a weighted average grade of $1.46 \%$ copper.

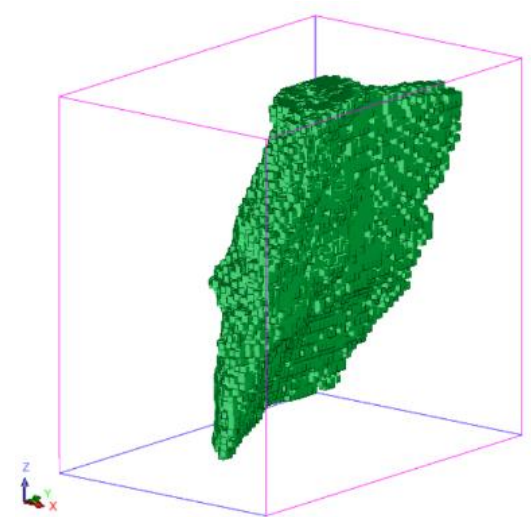

Figure 3 Isometric view of block model of the case study deposit

The IP optimisation framework is solved using a MATLAB environment (The MathWorks 2018) and the IBM ILOG CPLEX engine (IBM 2015) with a 5\% gap (the feasible integer solution found is proven to be within $5 \%$ of the optimal), on a $3.40 \mathrm{GHz}$ Intel Core i7 machine with $16 \mathrm{~GB}$ of RAM.

The dimensions considered for the column unit layout optimisation of each undercut sections are $20 \mathrm{~m}$ in the $X$ direction and $30 \mathrm{~m}$ in the $Y$ direction to mimic some common spacing measures of $10 \mathrm{~m}$ between drawpoints on the same drawbell and $20 \mathrm{~m}$ between different drawpoint excavations.

The operational and economic parameters considered are shown in Table 1.

Table 1 Operational and economical parameters for the application of the optimisation framework

\begin{tabular}{|c|c|c|c|}
\hline Parameter & Value & Unit & Description \\
\hline$T$ & 15 & Year & Number of periods (life of the mine) \\
\hline $\bar{M}$ & 1,200 & ktonne/period & Maximum mining capacity per period \\
\hline$\underline{M}$ & 500 & ktonne/period & Minimum mining capacity per period \\
\hline$\overline{D R}$ & 150 & ktonne/column/period & Maximum draw rate per column unit per period \\
\hline$\underline{D R}$ & 60 & ktonne/column/period & Minimum draw rate per column unit per period \\
\hline $\bar{H}$ & 300 & Metres & Maximum column height \\
\hline$\underline{H}$ & 100 & Metres & Minimum column height \\
\hline Recovery & 85 & $\%$ & Metal process recovery \\
\hline Price & 6,000 & $\$ /$ tonne & Selling price of the metal \\
\hline Cost & 26.1 & $\$ /$ tonne & Extraction cost (mining + processing) \\
\hline Discount rate & 10 & $\%$ & Discount rate \\
\hline a & 290 & $\circ$ & Main horizontal mining direction \\
\hline a1 & 45 & $\circ$ & Concave face angle \\
\hline a2 & 45 & $\circ$ & Concave face angle \\
\hline
\end{tabular}


The minimum draw rate from which the mining units are built on this test model was 60 ktonne/column/ period, which, considering the dimensions of the column units used, is roughly equivalent to drawing about 5 " $(12.7 \mathrm{~cm})$ per day from each column unit. This value is in line with some reported operational parameters. The optimisation model is run for an interval of levels between 30 (elevation $705 \mathrm{~m}$ ) and 45 (elevation 555 $\mathrm{m}$ ) from the block model $\mathrm{z}$ index. Figure 4 shows the NPV and metal content obtained by the optimisation model from each level.

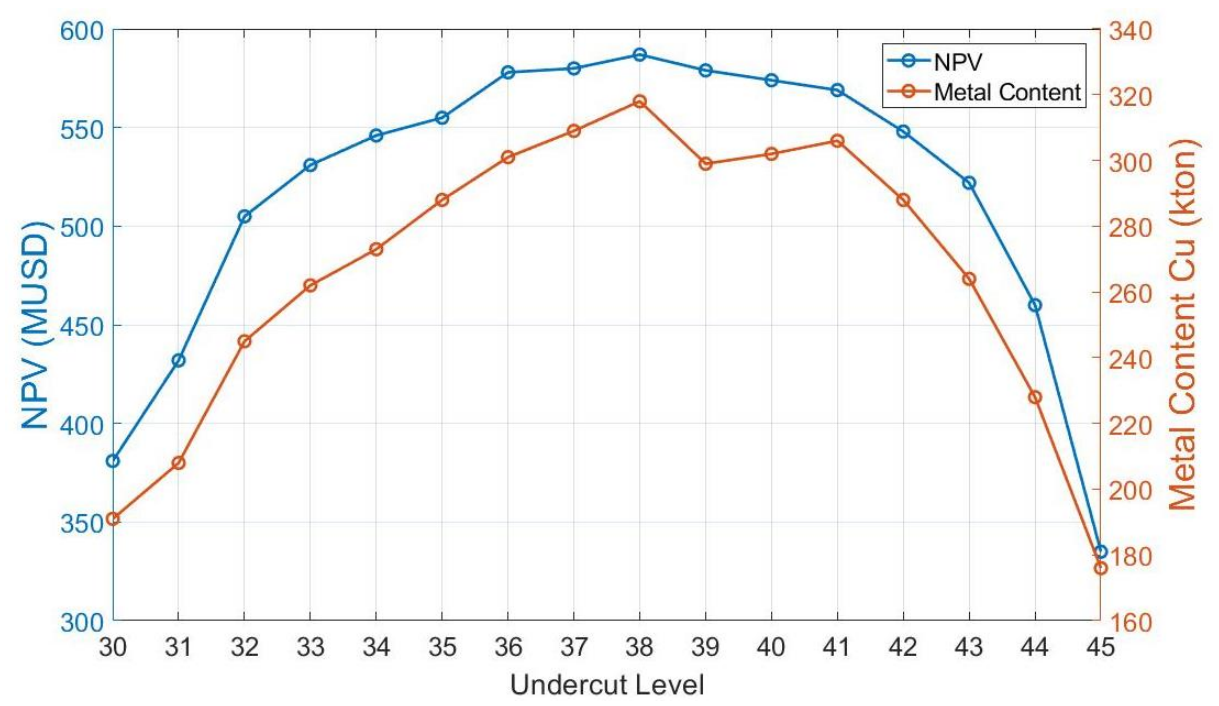

Figure 4 NPV and metal content obtained from the optimisation model for each level. Level 38 appears as the optimal undercut level

The optimisation model yielded level 38, at an elevation of $625 \mathrm{~m}$, as the optimal undercut elevation for the case study, with a NPV of USD $587.85 \mathrm{M}$ and a total of 318 ktonne of $\mathrm{Cu}$. This level is selected by analysing not only the total blocks above each undercut but by also considering operating constraints and a mining advancement direction, with the mining reserves and NPV obtained subjected to operational constraints. The undercut layout consists of 64 column units, which are representative of 128 drawpoints. Overall, the computing time for each level was about 30-60 minutes depending on the number of ore blocks in each undercut plan section. The undercut ore areas varied between 38,000 and $57,000 \mathrm{~m}^{2}$. On larger undercuts from massive deposits, the processing times are expected to be longer. However, an optimal solution can still be reached in a reasonable time.

Figure 5(a) shows the optimal layout for level 38. This layout maximises the metal content of the deposit based on the described aggregation of individual columns into the column units to represent the extraction practice in block caving, considering operational constraints. This layout also gives some insight into the potential drawpoint layout for detailed engineering studies at a later stage of the project.

Figure 5(b) shows the extraction opening sequence of the column units in the optimal undercut level. This sequence achieves the maximum NPV considering the horizontal concave mining direction. At the time of the development of this model, the starting point is selected to be the farthest column unit based on the direction advancement. 


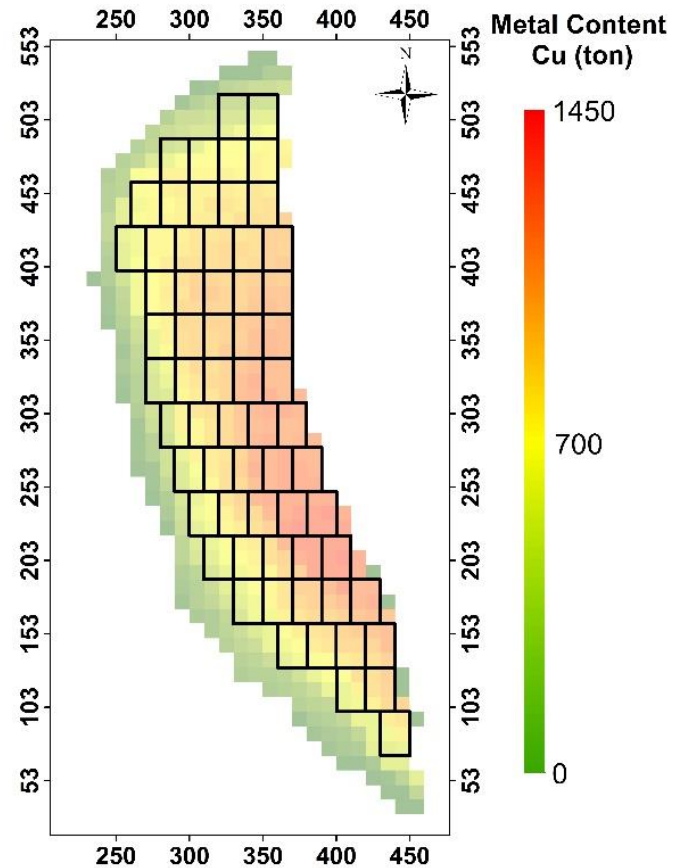

(a)

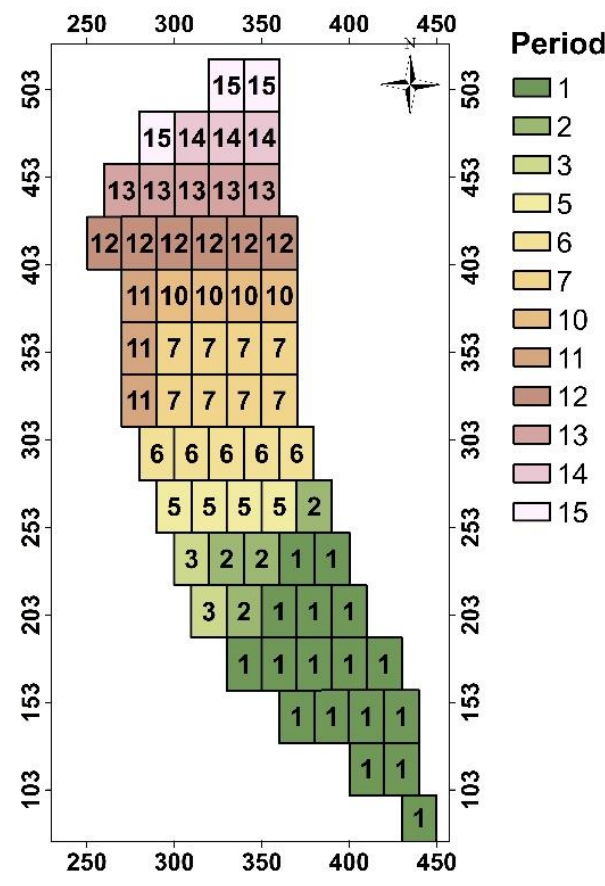

(b)

Figure 5 (a) Draw layout for optimal undercut level 38; and, (b) Sequence of columns opening to apply the horizontal discounting on the NPV at undercut level 38

One of the main concerns in mine planning is the risk of building an initial design based on a set of economic assumptions that are uncertain in the future. To deal with this uncertainty, a sensitivity analysis is carried out to understand the behaviour of a decision or design over multiple scenarios. The model proposed can be easily set up to evaluate multiple scenarios for any required economic or technical parameter.

As an example, a sensitivity analysis on the price of the metal is presented. The price of the metal is a decisive factor on the profitability over the life of a mine, but it is also one of the most uncertain parameters as it is dictated by global market conditions.

For the model presented, the response of the NPV and, most importantly, the most profitable level are evaluated over different price scenarios. The selection of an undercut elevation is not a flexible decision. Once development has started, it is impossible or very costly to start over. Therefore, it is important to evaluate multiple economic conditions when making a decision. There is also geotechnical uncertainty. However, the model takes as input parameters a starting point and mining direction as well as draw rates, all of which are assumed to be defined considering the proper geotechnical environment.

Table 2 shows the results of the sensitivity analysis on different prices. Two lower cases and two upper cases were considered at $10 \%$ intervals each. The model was set up to run on the same technical parameters as specified previously.

Table 2 Sensitivity analysis for different price scenarios

\begin{tabular}{llllll}
\hline & \multicolumn{2}{l}{ Lower cases } & \multicolumn{2}{l}{ Base case } & \multicolumn{2}{l}{ Upper cases } \\
\hline Variation (\%) & -20 & -10 & - & +10 & +20 \\
Price (\$/tonne) & 4,800 & 5,400 & 6,000 & 6,600 & 7,200 \\
Optimum level & 39 & 38 & 38 & 38 & 38 \\
NPV & 327.34 & 430.95 & 587.85 & 712.32 & 824.61 \\
\hline
\end{tabular}


The optimum level remains at level 38, at an elevation of $625 \mathrm{~m}$, for both the upper cases and up to a $-10 \%$ variation in the price. This means that starting the operation with the undercut placed at that level will maximise the profitability of the mine even over optimistic scenarios, and with metal price value reductions of up to $10 \%$. The optimum level does change when a $20 \%$ reduction in the copper price is considered, and moves up to level 39, at an elevation of $635 \mathrm{~m}$.

In a pessimistic scenario, with the price set at $\$ 4,800$ per tonne, a better NPV while scheduling based on the mining direction is found at a different level. However, setting the undercut at level 38 seems to be a robust decision, as it maximises the NPV over multiple price scenarios and would likely be the most profitable decision for this case study.

\section{Conclusion}

The optimisation framework achieves an optimal solution in under 20 minutes for each level and gives insight into the possible alternatives for footprint layout design and development sequence. The fast processing times allow for an iterative process to find the most profitable undercut elevation considering operational constraints and guaranteeing an optimal solution via an IP model. While the assumptions used to simplify the problem cause a loss of resolution for the long-term scheduling of the caving mine, the final goal of this model is not to find an optimal schedule, but to include the mining direction discounting on the NPV evaluation for the selection of an undercut elevation.

Although it would be expected for the computing time to increase for massive deposits with a larger footprint area, the model could still perform in reasonable timeframes. In this particular case, with a footprint of $57,000 \mathrm{~m}^{2}$, the optimal solution was reached after 20 minutes of processing, and the iterating process over multiple levels is a computational parallelisable process.

The model could be still improved by considering dilution mixing models such as Laubscher's (non-linear mixing models would require another type of mathematical formulation), considering grade uncertainty via multiple geostatistical simulations of the deposit, and considering specific starting points for the extraction sequence. Multiple metals can also be considered when building the objective function, that is, when calculating the economic block values. Considering multiple economic metals, common in many porphyry deposits suitable to block caving, would not add to the computing times.

The mining direction and starting point in the sequence still play a major role in the NPV of a caving project. The model takes as an input a mining direction, usually defined considering the geotechnical conditions of the deposit, and evaluates the profitability of setting the undercut level at different elevations considering the economic discounting from this direction. Once an elevation or a small number of undercut levels have been found to yield the highest NPV for the deposit, the model could be applied to evaluate different mining directions and scenarios.

The initial layout optimisation step is also of interest as it could be improved to account for the different geometrical constraints of the common drawpoint layout schemes, and possible underground infrastructure placement, in order to develop a tool to optimise the drawpoint locations in the caving footprint.

\section{Acknowledgement}

The authors thank the Mining Optimisation Laboratory (MOL) of the University of Alberta.

\section{References}

Bartlett, P 1992, 'The design and operation of a mechanized cave at Premier Diamond Mine', in HW Glen (ed.), Proceedings of MassMin 1992, The South African Institute of Mining and Metallurgy, Johannesburg.

Brown, ET 2002, Block Caving Geomechanics, Julius Kruttschnitt Mineral Research Centre, Indooroopilly, and The University of Queensland, Brisbane.

Chanda, E 1990, 'An application of integer programming and simulation to production planning for a stratiform ore body', Mining Science and Technology, vol. 11, no. 2, pp. 165-172. 
Dassault Systèmes 2018, GEMS PCBC, computer software, Dassault Systèmes, Paris, https://www.3ds.com/products-services/geovia /products/gems

Diering, T 2000, 'PC-BC: a block cave design and draw control system', in G Chitombo (ed.), Proceedings of MassMin 2000, The Australasian Institute of Mining and Metallurgy, Melbourne.

Diering, T, Richter, O \& Villa, D 2008, 'Block cave production scheduling using PCBC', in H Schunnesson \& E Nordlund (eds), Proceedings of MassMin 2008, Luleå University of Technology, Luleå.

Elkington, TB, Richter, L \& Richter, O 2012, 'Block caving outline optimisation', Proceedings of MassMin 2012, Canadian Institute of Mining, Metallurgy and Petroleum, Westmount.

IBM 2015, IBM ILOG CPLEX Optimization Studio, computer software, IBM, Armonk, https://www.ibm.com/au-en/marketplace/ibmilog-cplex

Khodayari, F \& Pourrahimian, Y 2015, 'Mathematical programming applications in block-caving scheduling: a review of models and algorithms', International Journal of Mining and Mineral Engineering, vol. 6, no. 3, pp. 234-257.

Laubscher, D 1994, 'Cave mining: state of the art', Journal of the South African Institute of Mining and Metallurgy, vol. 94, no. 10, pp. 279-293.

Malaki, S, Khodayari, F, Pourrahimian, Y \& Liu, W 2017, 'An application of mathematical programming and sequential Gaussian simulation for block cave production scheduling', in Y Potvin (ed.), Proceedings of the First International Conference on Underground Mining Technology, Australian Centre for Geomechanics, Perth, pp. 323-338.

Pourrahimian, Y \& Askari-Nasab, H 2014, 'An application of mathematical programming to determine the best height of draw in block-cave sequence optimisation', Mining Technology, vol. 123, no. 3, pp. 162-172.

Pourrahimian, Y, Askari-Nasab, H \& Tannant, D 2012, 'Block caving production scheduling optimization using mathematical programming', Proceedings MassMin 2012, Canadian Institute of Mining, Metallurgy and Petroleum, Westmount.

Pourrahimian, Y, Askari-Nasab, H \& Tannant, D 2013, 'A multi-step approach for block-cave production scheduling optimisation', International Journal of Mining Science and Technology, vol. 23, no. 5, pp. 739-750.

Rahal, D, Dudley, J \& Hout, G 2008, 'Developing an optimised production forecast at Northparkes E48 mine using MILP, in H Schunnesson \& E Nordlund (eds), Proceedings of MassMin 2008, Luleå University of Technology, Luleå.

Rubio, E 2002, 'Long term planning of block caving operations using mathematical programming tools', MSc thesis, The University of British Columbia, Vancouver.

The MathWorks 2018, MATLAB, version R2018a, computer software, The MathWorks, Natick, https://au.mathworks.com/products /matlab.html

Tukker, H, Holder, A, Swarts, B, van Strijp, T \& Grobler, E 2016, 'The CCUT block cave design for Cullinan Diamond Mine', Journal of the Southern African Institute of Mining and Metallurgy, vol. 116, no. 8, https://dx.doi.org/10.17159/2411-9717/2016/v116n8a2

Vargas, E, Morales, N \& X, Emery 2014, 'Footprint and economic envelope calculation for block/panel caving mines under geological uncertainty', in R Castro (ed), Proceedings of the Third International on Block and Sublevel Caving, University of Chile, Santiago, pp. 449-456. 